\title{
UMA CONEXÃO ENTRE ALGAS E NUVENS: FUNDAMENTOS TEÓRICOS DA HIPÓTESE CLAW E SUAS IMPLICAÇÕES PARA AS MUDANÇAS CLIMÁTICAS
}

\author{
Nei Freitas Nunes-Neto ${ }^{1,2 *}$, Ricardo Santos do Carmo ${ }^{1,3}$ \& Charbel Niño El-Hani ${ }^{1,2,3}$ \\ ${ }^{1}$ Universidade Federal da Bahia (UFBA), Instituto de Biologia, Departamento de Biologia Geral, Grupo de Pesquisa em História, Filosofia e Ensino de \\ Ciências Biológicas. Rua Barão de Jeremoabo, s/n, Campus Universitário de Ondina, Salvador, Bahia, Brasil. CEP: 40170-115. \\ ${ }^{2}$ Universidade Federal da Bahia (UFBA), Instituto de Biologia, Programa de Pós-Graduação em Ecologia e Biomonitoramento. Rua Barão de Jeremoabo, \\ s/n, Campus Universitário de Ondina, Salvador, Bahia, Brasil. CEP: 40170-115. \\ ${ }^{3}$ Universidade Federal da Bahia (UFBA)/Universidade Estadual de Feira de Santana (UEFS), Instituto de Física, Programa de Pós-Graduação em \\ Ensino, Filosofia e História das Ciências. Rua Barão de Jeremoabo, s/n, Campus Universitário de Ondina, Salvador, Bahia, Brasil. CEP: 40170-115. \\ E-mails: nunesneto@gmail.com(*),rscarmo@ufba.br, charbel@ufba.br
}

\section{RESUMO}

Neste trabalho, abordamos o surgimento e o desenvolvimento de uma hipótese científica sobre uma conexão entre algas e nuvens, de grande relevância para a compreensão das mudanças climáticas, e tecemos ainda algumas considerações epistemológicas. Foram importantes para o surgimento da hipótese investigações empreendidas por Lovelock e colaboradores, no início da década de 1970, em busca de um intermediário estável do ciclo do enxofre, que seria responsável pelo transporte deste elemento dos oceanos para a terra. A partir de estudos sobre a liberação de sulfeto de dimetila (DMS) por espécies do fitoplâncton marinho e sobre processos de formação de nuvens e sua relação com o albedo planetário, Charlson e colaboradores propuseram, em 1987, que a rápida oxidação do DMS levaria à formação de um aerossol ( $\mathrm{NSS}_{-} \mathrm{SO}_{4}{ }^{2-}$ ), que, ao ser oxidado, formaria núcleos necessários para a condensação de vapor d'água e conseqüente formação de nuvens sobre os oceanos. Como as nuvens refletem parte da radiação solar que chega ao planeta, elas contribuem para o resfriamento da superfície planetária. Por esta razão, o DMS tem sido apontado como um importante gás estufa negativo, que poderia contrabalançar os efeitos de aquecimento de gases estufa como $\mathrm{CO}_{2}$ e $\mathrm{CH}_{4}$. Deve-se apontar, contudo, que o grau em que o DMS pode contribuir para o resfriamento da superfície planetária ainda é uma questão aberta na comunidade científica. A hipótese proposta por Charlson e colaboradores tem exercido, ainda assim, grande impacto nas pesquisas sobre mudanças climáticas, tendo dado origem a toda uma nova área de pesquisas científicas interdisciplinares, conhecida como 'conexão algas-nuvens'. Aqui, buscaremos mostrar as implicações desta hipótese e do novo campo de investigação por ela gerada para os estudos sobre mudanças climáticas, bem como sobre as relações entre a biota e seu ambiente físico-químico, um tema central da Ecologia.

Palavras-chave: Hipótese CLAW, conexão algas-nuvens, sulfeto de dimetila, biogeoquímica do enxofre, mudanças climáticas.

\footnotetext{
ABSTRACT

A LINK BETWEEN ALGAE AND CLOUDS: THEORETICAL GROUNDS OF THE CLAW HYPOTHESIS AND ITS IMPLICATIONS TO CLIMATE CHANGE. In this work, we address the origins and development of a scientific hypothesis about a connection between algae and clouds that is greatly relevant to the understanding of climate change, advancing also some epistemological remarks. Studies carried out by Lovelock and colleagues in the beginnings of the 1970s were important to the construction of this hypothesis. Those studies searched for a stable intermediary in the sulphur cycle, which would be responsible for transferring this element from oceans to land. Based on studies about the release of dimethyl sulphide (DMS) by marine phytoplankton species and the process of cloud formation, as well as its relationship with planetary albedo, Charlson and colleagues proposed in 1987 that the rapid oxidation of DMS would lead to the formation of an
} 
aerosol (NSS- $\mathrm{SO}_{4}{ }^{2-}$ ), which, when oxidized, would constitute nuclei required for the condensation of water vapor, and, thus, to cloud formation over the oceans. Since clouds reflect part of the solar radiation that reaches the planet, they contribute to cool the planetary surface. Therefore, DMS has been pointed out as a negative greenhouse gas, which could counterbalance the heating effects of greenhouse gases such as $\mathrm{CO}_{2}$ and $\mathrm{CH}_{4}$. We should stress, however, that the degree in which DMS can contribute to cool the Earth surface is still an open issue in the scientific community. Charlson and colleagues' hypothesis has nonetheless exerted a great impact on researches about climate change, giving rise to a whole new area of interdisciplinary scientific research, known as the 'cloud-algae link'. Here, we intend to show how this hypothesis and the new research field resulting from it have an impact on studies about climate change, and, also, about the relationships between the biota and its physicochemical environment, a central theme of Ecology.

Keywords: CLAW hypothesis, cloud-algae link, dimethyl sulphide, sulphur biogeochemistry, climate change.

\section{RESUMEN}

UNA CONEXIÓN ENTRE ALGAS Y NUBES: FUNDAMENTOS TEÓRICOS DE LA HIPÓTESIS CLAW Y SUS IMPLICACIONES PARA LOS CAMBIOS CLIMÁTICOS. Este artículo aborda el surgimiento y desarrollo de una hipótesis científica sobre una conexión entre algas y nubes, de gran relevancia para la comprensión de los cambios climáticos, haciendo consideraciones epistemológicas a este respecto. El origen de esta hipótesis esta asociada a los estudios realizados por Lovelock y colaboradores en el inicio de los años 1970, en una búsqueda por un intermediario estable en el ciclo del azufre, que sería responsable por el transporte de este elemento de los océanos para la tierra. A partir de estudios sobre la liberación del sulfuro de dimetilo (DMS) por especies del fitoplancton marino y sobre procesos de formación de nubes y su relación con el albedo planetario, Charlson y colaboradores hicieran en 1987 la propuesta de que la rápida oxidación de DMS llevaría a la formación de un aerosol ( $\mathrm{NSS}_{-} \mathrm{SO}_{4}{ }_{4}^{2-}$ ), que, cuando oxidado, formaría los núcleos necesarios para la condensación del vapor de agua y consecuente formación de nubes sobre los océanos. Las nubes refleten parte de la radiación solar que llega al planeta y, luego contribuyen para la refrigeración de la superficie planetaria. Así, se apunta que el DMS es un importante gas de efecto invernadero negativo, que podría actuar como un contrapeso a los efectos del calentamiento de gases como $\mathrm{CO}_{2} \mathrm{y} \mathrm{CH}_{4}$. Sin embargo, es necesario decir que el grado en que el DMS puede contribuir para la refrigeración de la superficie planetaria es todavia una cuestión abierta para la comunidad científica. La hipótesis propuesta por Charlson y colaboradores ha ejercido gran impacto en las investigaciones sobre los cambios climáticos, originando una área enteramente nueva de investigaciones interdisciplinarias llamada 'conexión algas-nubes'. En este artículo, buscaremos mostrar las implicaciones de esta hipótesis y del nuevo campo de investigaciones que ha generado ideas para los estudios sobre los cambios climáticos, así como sobre las relaciones entre la biota y el ambiente físico-químico, un tema central de la ecología.

Palabras clave: Hipótesis CLAW, conexión algas-nubes, sulfuro de dimetilo, biogeoquímica del azufre, cambios climáticos.

\footnotetext{
"Até mesmo se CLAW vier a ser totalmente irrelevante em termos climáticos, ela deixará um legado rico e fundamentalmente importante quanto às nossas percepções de como o mundo funciona e ao modo como precisamos fazer nossa ciência para entendê10" (árbitro anônimo, citado em Liss \& Lovelock 2007, p. 378).
}

\section{INTRODUÇÃO}

A epígrafe deste artigo é uma citação ipsis litteris de um árbitro protegido pelo anonimato e, precisamente por essa razão, coloca em destaque o argumento central que pretendemos defender aqui. Nosso objetivo neste trabalho é analisar o desenvolvimento de uma hipótese científica que, com pouco mais de duas décadas de existência, exerceu e vem exercendo grande impacto sobre as pesquisas relacionadas à 
compreensão da dinâmica planetária do enxofre e das mudanças climáticas na Terra. A hipótese em questão ficou conhecida na literatura científica como 'hipótese CLAW', um acrônimo dos nomes de seus quatro cientistas proponentes (Charlson, Lovelock, Andreae e Warren). Ela foi apresentada à comunidade científica em 1987 e propõe, em linhas gerais, um mecanismo de retroalimentação (feedback) negativo no qual algas planctônicas exercem influência no clima global pela síntese e exsudação de um composto de enxofre. $\mathrm{Na}$ atmosfera, esse composto sofre oxidação e origina partículas com propriedades físicas e químicas favoráveis à condensação de água na troposfera, onde as nuvens formadas refletem para o espaço uma fração significativa da radiação solar incidente. Em outras palavras, este é um mecanismo de retroalimentação, de longo termo, envolvendo em escala global o fitoplâncton marinho e o clima da Terra. A comunidade científica de fins da década de 1980 recebeu bem essa hipótese e hoje ela tem atraído interesse substancial de cientistas de diferentes disciplinas (ex. Física e Geofísica atmosférica, Química e Geoquímica atmosférica, Biogeoquímica, Taxonomia, Oceanografia e Biologia evolutiva), que trabalham num esforço conjunto para lançar luz sobre os modos como os seres vivos interagem com o ambiente físico-químico.

Nas palavras de Huebert (2007, p. 375), "a hipótese CLAW revolucionou a maneira como pensamos o sistema Terra", um reconhecimento ao fato de que, entre o final da década de 1980 e o início dos anos 1990, essa hipótese científica conduziu ao desenvolvimento de um novo e interessante campo de pesquisas interdisciplinares conhecido como 'conexão algas-nuvens' (cloud-algae link). Essa designação atual é bastante similar a outra mais antiga, algaecloud connection, proposta por Andrew Bell em 1986. Ainda que Bell (1986) tenha praticamente nomeado o que se constituiria um interessante e promissor campo de pesquisas, a hipótese de um mecanismo de retroalimentação negativo entre as algas marinhas e o clima foi uma contribuição genuína de Charlson et al. (1987). Nesse campo, de acordo com Ayers \& Cainey (2007), a orientação explícita é elucidar as relações que integram os processos biológicos, físicos e químicos no sistema Terra.

Este artigo está estruturado da seguinte maneira. A próxima seção tem início com uma questão científica importante na década de 1970, a saber: qual o composto que transporta o enxofre dos oceanos para as massas de terra e, assim, completa uma lacuna então existente no ciclo deste elemento? A análise desta questão é importante porque nela estão os antecedentes da hipótese CLAW. Ainda nesta seção, apresentamos em mais detalhes o mecanismo proposto pela hipótese, assim como seus fundamentos epistemológicos. A partir do exposto nesta seção, apresentamos na seção subseqüente as principais implicações da hipótese CLAW para a compreensão das relações dinâmicas entre os seres vivos e o ambiente físico-químico em escala global. A partir dos argumentos expostos, lançamos nossas considerações finais na última seção.

\section{DE UMA LACUNA NO CICLO DO ENXOFRE A UM NOVO CAMPO DE PESQUISA INTERDISCIPLINAR}

Até o início da década de 1970, havia muitas dúvidas acerca da dinâmica do ciclo do enxofre. Em particular, uma lacuna importante em nosso conhecimento dizia respeito a qual seria o composto químico estável que atuaria como transportador de enxofre dos oceanos para a terra (Lovelock 1991). Em condições naturais, o enxofre existe em diversos estados de oxidação e participa de reações que têm importantes conseqüências geoquímicas. Nos organismos vivos, está tipicamente no estado bivalente, ligado ao carbono e hidrogênio, formando dois aminoácidos essenciais: cisteína e metionina (Nelson \& Cox 2009). Apesar de ser um elemento essencial para todos os organismos, o enxofre é mais abundante nos oceanos (comumente na forma de íons sulfato [ $\mathrm{SO}_{4}^{2-}$ ] livres) do que nos continentes (Lovelock 1991, Charlson et al. 2000). A ação mecânica da água ao longo dos rios, que provoca a lixiviação dos solos, e a intemperização das rochas (sobretudo, as sedimentares) resultam numa água cada vez mais rica em enxofre na forma de íons sulfato (quanto mais próximo da foz). Na medida em que é um elemento essencial, mas que não é um fator limitante para os organismos nos ecossistemas terrestres, a seguinte questão foi posta: qual mecanismo repõe o enxofre nas massas de terra? Entendemos hoje, de acordo com Stallard (2000), que os processos de intemperização exercem grande influência na regulação do ciclo dos 
elementos na atmosfera e nos oceanos, e uma das vias de retorno do enxofre aos continentes é o ciclo das rochas. No começo dos anos 1970, entretanto, o fluxo e as transformações físicas e químicas do enxofre nos principais reservatórios eram pouco compreendidos, suscitando o interesse de vários cientistas (Lovelock 1997).

A visão convencional daquela época atribuía ao sulfeto de hidrogênio $\left(\mathrm{H}_{2} \mathrm{~S}\right)$ o papel de composto químico responsável pela transferência do enxofre dos oceanos para a terra. Um requisito necessário para apoiar empiricamente esta hipótese era que grandes quantidades de $\mathrm{H}_{2} \mathrm{~S}$ fossem liberadas dos oceanos, como uma maneira de compensar a perda de enxofre do ambiente terrestre. Entretanto, como notou o químico inglês James Lovelock, a água do mar é muito oxidante para permitir a existência de concentrações de $\mathrm{H}_{2} \mathrm{~S}$ que sejam suficientes para repor o enxofre aos ecossistemas terrestres. Além disso, o $\mathrm{H}_{2} \mathrm{~S}$ possui um odor muito forte e característico, o que o tornaria facilmente detectável (Lovelock 1991). Contudo, esse composto não foi encontrado, o que abriu espaço para novas conjecturas.

Em 1972, apoiando-se num trabalho do químico e bioquímico inglês Frederick Challenger, o qual observou que muitas algas marinhas emitem sulfeto de dimetila (doravante, DMS), Lovelock et al. (1972) colocaram em xeque a visão tradicional e propuseram que o "DMS é o composto natural de enxofre que cumpre o papel originalmente atribuído ao $\mathrm{H}_{2} \mathrm{~S}$; aquele de transferir o enxofre dos mares através do ar para as superfícies de terra" (Lovelock et al. 1972, p. 452).

É importante notar que a sugestão de que o DMS seria o intermediário envolvido na dinâmica planetária do enxofre surge no âmbito da teoria Gaia, desenvolvida desde a década de 1960 por Lovelock e outros cientistas, como a microbióloga norte-americana Lynn Margulis. De acordo com a teoria Gaia, todos os seres vivos estão profunda e sistematicamente envolvidos em mecanismos de regulação das variáveis físico-químicas do sistema Terra, freqüentemente atuando como fontes de compostos químicos que podem ser detectados, por exemplo, na atmosfera (Lovelock 1988). Cabe notar que esta idéia, de uma influência poderosa da vida sobre o ambiente físico da Terra, aparece pela primeira vez na teoria da Biosfera, elaborada, a partir da década de 1920, pelo cientista russo-ucraniano Vladimir Ivanovitch Vernadsky (Vernadsky 2007). Entretanto, foi apenas entre as décadas de 1960 e 1970, com aportes teóricos advindos da cibernética, de estudos sobre detecção de vida fora da Terra e o uso de ferramentas de modelagem matemática (Lovelock 1991), que a idéia vernadskyana pôde ser colocada com mais precisão, o que significa que a teoria Gaia não é um plágio da teoria da Biosfera, como afirmam Levit \& Krumbein (2000). Neste sentido, a herança de Vernadsky foi colocada a partir da década de 1960 em novas bases, que possibilitaram a criação de modelos testáveis, como o Daisyworld (ver Watson \& Lovelock 1983) e o modelo proposto pela hipótese CLAW (para outros modelos no âmbito da Ciência do Sistema Terra, ver Kump et al. 1999, Jacobson et al. 2000, Nunes-Neto 2008, Carmo et al. 2009).

Após a publicação do artigo de Lovelock et al. (1972), trabalhos subseqüentes forneceram dados sobre a distribuição global e concentração de DMS nos oceanos. Andreae e Raemdonck (1983), por exemplo, estimaram o fluxo desse composto na interface oceano-atmosfera, Bigg et al. (1984) investigaram os mecanismos de produção de partículas a partir do DMS e, recentemente, Kasting \& Siefert (2002) e Spiese et al. (2009) mostraram que várias espécies de algas marinhas liberam DMS para a atmosfera. O DMS é reconhecidamente a fonte biológica dominante desse composto volátil de enxofre para a atmosfera marinha, originando partículas necessárias à formação de nuvens. No entanto, é relevante destacar que a presença dessas partículas na troposfera não está limitada ao enxofre derivado do DMS liberado pelas algas marinhas (Cainey et al. 2007); há evidências recentes (Clarke et al. 1999, Shaw 2007, Liss \& Lovelock 2007) de nucleação direta na interface oceano-atmosfera a partir de sais marinhos e de partículas orgânicas, tipicamente vírus (Fuhrman 1999), presentes em 50-90\% dos aerossóis de sulfato (Leck \& Bigg 2007).

Seja como for, está bem estabelecido que os oceanos estão supersaturados em DMS em relação às concentrações atmosféricas (Barnard et al. 1982, Liss et al. 1993) e um conjunto de evidências empíricas tem revelado que o DMS cumpre um importante papel no processo de indução à formação de nuvens. Por essa razão, a proposta de Lovelock et al. (1972) contribuiu em termos teóricos e empíricos para o 
desenvolvimento de um campo de pesquisas cada vez mais vigoroso, a Ciência do Sistema Terra (CST). $\mathrm{E}$, além disso, ela foi incorporada à biogeoquímica, figurando hoje como parte do conhecimento científico convencional sobre a Terra (Kump et al. 1999, Jacobson et al. 2000).

Numa abordagem histórica da hipótese CLAW, é preciso deixar claro que a relação entre a refletividade das nuvens e o clima já havia sido imaginada por Twomey (1977) e, quatro anos antes da proposta de Charlson et al. (1987), Shaw (1983) já havia proposto que aerossóis atmosféricos de enxofre provenientes da biota marinha poderiam participar do balanço radiativo do planeta, afetando significativamente $o$ clima. Contudo, nenhuma dessas propostas alcançou uma compreensão satisfatória dos processos de integração entre algas planctônicas, compostos de enxofre, nuvens e clima, como Charlson et al. (1987) fizeram. Nesse artigo, baseando-se numa série de trabalhos prévios sobre química e física atmosférica, microfísica de nuvens, bioquímica oceânica, ciclagem do enxofre, e apoiando-se numa herança vernadskyana - de influência poderosa, construtora, dos organismos sobre seu ambiente -, os autores propuseram que a rápida oxidação do DMS na atmosfera produz "partículas aerossol sulfato de sal não-marinho" (non-sea-salt sulphate aerosol particles) que funcionam como núcleos de condensação de vapor d'água, contribuindo para a formação de nuvens sobre os oceanos. Esses núcleos são denominados 'núcleos de condensação de nuvens' (NCN; em inglês CCN, cloud condensation nuclei).

De modo muito breve e esquemático, a hipótese CLAW propõe um mecanismo de retroalimentação negativo envolvendo fitoplâncton, DMS, NCN e nuvens, com implicações climáticas em escala global. De acordo com Charlson et al. (1987, p. 656), as regiões oceânicas mais quentes, mais salinas e que são mais intensamente iluminadas têm as maiores taxas de emissão de DMS para a atmosfera. Evidências para a influência da temperatura sobre a emissão de DMS advêm, dentre outros, dos trabalhos de Bopp et al. (2003) e Gabric et al. (2004). Estes trabalhos, estudos de modelagem em escala global, mostraram que há um pequeno aumento na produção de DMS em resposta a um aquecimento do oceano, resultante da concentração crescente de $\mathrm{CO}_{2}$ atmosférico. Assim, as porções de água nos oceanos que não estão cobertas por nuvens tendem a ser mais iluminadas e se aquecer mais, já que recebem a radiação solar diretamente. $\mathrm{O}$ aumento da temperatura deve levar a um aumento da produção de DMS pelas algas, contribuindo para a formação de mais nuvens sobre os oceanos (Lovelock 1997). As nuvens têm um albedo alto e reduzem a temperatura e a luminosidade da superfície, porque refletem grande fração da radiação solar incidente. Portanto, há uma redução da quantidade de radiação solar que alcança a superfície da água, levando a uma diminuição da temperatura nesta região, com conseqüente diminuição na produção de DMS e nuvens e, novamente, a um aumento da incidência de raios solares sobre a superfície da água, o que fecha a alça de retroalimentação. A Figura 1 fornece uma visão geral do mecanismo proposto pela hipótese CLAW.

A rigor, devemos salientar, as algas planctônicas não sintetizam o DMS, mas sim o seu precursor, o dimetilsulfoniopropionato (DMSP) (Charlson et al. 1987, Andreae \& Crutzen 1997, Lovelock 1991, Simó 2001). Pensava-se anteriormente que o DMSP estava restrito ao meio intracelular, por não poder atravessar a membrana plasmática sem um transportador específico (Kiene et al. 1998). No entanto, foi recentemente descoberto que uma fração pequena do DMSP é exsudada pela célula para o ambiente marinho (Simó 2001). Mecanismos de liberação do DMSP para o exterior são a autólise, a lise celular por ataque viral, além da herbivoria (Simó 2001). A produção de DMS depende da atividade da enzima DMSP-liase; a demetilação enzimaticamente mediada do DMSP origina o DMS que é liberado para a água do mar quando as algas morrem (Simó \& Pedros-Alió 1999). Nos oceanos, há uma notável diversidade de vírus marinhos (Suttle et al. 1991, Proctor \& Fuhrman 1991, Proctor 1997) capazes de infectar praticamente todo tipo de fitoplâncton, possivelmente participando da regulação populacional desse grupo (Fuhrman 1999). O fitoplâncton é base da rede alimentar marinha, sendo formado tanto por procariotos (ex., as cianobactérias Prochlorococcus e Synechococcus) quanto por eucariotos (ex., as diatomáceas Thalassiosira pseudonana e T. oceanica, os dinoflagelados Karenia brevis e Prorocentrum minimum, e as haptófitas Isochrysis galbana e Emiliana huxleyi), freqüentemente identificados em florações correlacionadas ao fluxo de DMS para a atmosfera.

Entre esses organismos planctônicos, a espécie 
cocolitófora Emiliana huxleyi merece particular atenção porque tem a maior distribuição oceânica, é dominante nas florações (Brown \& Yoder 1994), principalmente em latitude média, e sintetiza muito DMSP (Keller 1991, Lovelock 1991). A predação de E. huxleyi pelo zooplâncton (ex., o protozoário Oxyrrhis marina) acaba por ser uma fonte substancial de DMS para a atmosfera, na medida em que a presença da DMSP-liase no interior do zooplâncton converte o DMSP em DMS (Wolfe et al. 1997). Além disso, devemos considerar as infecções virais como uma das interações ecológicas que são parte da dinâmica complexa do mecanismo de retroalimentação proposto pela hipótese CLAW, porque elas são um fator causal de liberação de DMS para a atmosfera. Em pelo menos um experimento de mesocosmos (Bratbak et al. 1993), as infecções responderam pela mortalidade de $25-100 \%$ de E. huxleyi numa floração. É tendo em conta essas interações ecológicas que se diz que o DMSP é um produto do fitoplâncton e o DMS é um produto do ecossistema (Cropp \& Norbury 2007). As interações ecológicas no ambiente marinho são, portanto, uma peça-chave para a nossa compreensão do mecanismo de retroalimentação proposto pela hipótese CLAW e, de fato, temos aprendido cada vez mais sobre elas; os resultados de DeBose et al. (2008), por exemplo, indicam que o DMSP sintetizado por algas bentônicas e fitoplâncton associadas aos recifes de corais funciona como sinalizador químico, atraindo peixes planctófagos. Apesar de sabermos

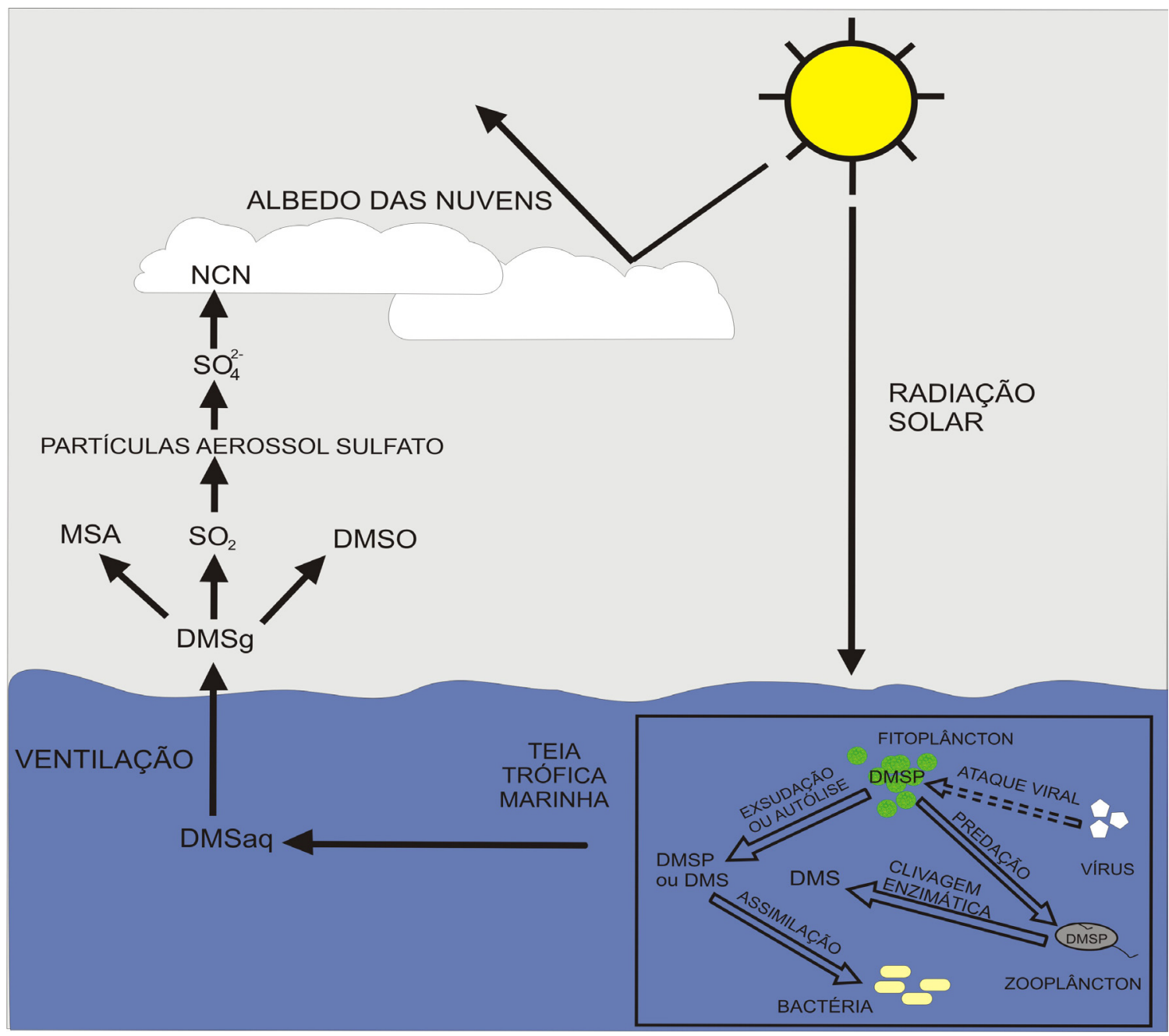

Figura 1. Representação esquemática dos principais mecanismos envolvendo a produção do DMS pela teia trófica marinha e sua rota no oceano e na atmosfera, contribuindo para a produção de nuvens. DMS : sulfeto de dimetila na água marinha; DMS : sulfeto de dimetila na atmosfera; DMSP: dimetilsulfoniopropionato; DMSO: dimetilsulfóxido; $\mathrm{MSA}$ : ácido metano sulfônico; $\mathrm{SO}_{2}$ : dióxido de enxofre; $\mathrm{SO}_{4}{ }^{2-}$ : íons sulfato; $\mathrm{NCN}$ : núcleos de condensação de nuvens. Para mais explicações, ver o texto. Figura elaborada pelos autores.

Figure 1. Schematic representation of the main mechanisms involved in the production of dymethyl sulphide by the marine biota and its route through sea and atmosphere leading to cloud formation. DMSaq: marine dymethyl sulphide; DMSg: atmospheric dymethyl sulphide; DMSP: dimethylsulfoniopropionate; DMSO: dimethyl sulfoxide; $\mathrm{MSA}$ : metano-sulfonic acid; $\mathrm{SO}_{2}:$ sulfuric dioxide; $\mathrm{SO}_{4}{ }^{2-}:$ sulfate ions; $\mathrm{NCN}$ : cloud condensation nuclei. See the text for further details. Figure elaborated by the authors. 
mais sobre essas interações, não temos ainda clareza sobre quais fatores levam às mudanças sazonais na biomassa e composição de espécies nas florações algais em condições naturais. Um fator-chave são as interações com zooplâncton, vírus e parasitas envolvendo inclusive competição por nutrientes mas há quem as minimize (ex., Smetacek \& Cloern 2008), destacando que as algas têm ciclos de vida selecionados numa escala de tempo ampla, regulados por fatores internos e externos, como geomorfologia e hidrologia local.

Nesse contexto, é importante considerar que a produção de DMS pelo fitoplâncton não deve ser interpretada como um ato de altruísmo global, com o suposto objetivo de manter a temperatura da Terra em valores adequados para a sobrevivência de toda a biota. O DMS é apenas um produto colateral do metabolismo das algas em interações com outros organismos da teia trófica marinha. Por sua vez, a síntese do DMSP - o precursor do DMS - tem custos metabólicos para as algas e, portanto, estes custos devem ser compensados com benefícios próprios. Com efeito, há evidências de que o DMSP cumpre, no mínimo, quatro papéis funcionais na célula: (1) é um soluto que contribui para o equilíbrio osmótico celular (Kirst 1996, Stefels 2000); (2) um antioxidante (Sunda et al. 2002); (3) um inibidor da cisteína e da metionina (através de um mecanismo de superabundância [overflow mechanism], cf. Stefels 2000, p. 192); e (4) um sinalizador químico na ecologia marinha e uma defesa química de espécies de algas contra predadores (Wolfe et al. 1997, Stefels 2000, Steinke et al. 2002). Essas quatro funções fisiológicas parecem ser importantes para o sucesso reprodutivo das algas e, por isso, trata-se de um mecanismo fisiológico que pode ter sido selecionado positivamente (ver Hamilton \& Lenton 1998 para uma boa abordagem evolutiva da síntese de DMSP).

Os resultados empíricos gerados desde Charlson et al. (1987) não apontam todos numa mesma direção, a favor ou contra o mecanismo de retroalimentação negativo proposto pela hipótese CLAW.Éamplamente aceito que a concentração de DMSP nos oceanos é um fator dependente da temperatura, mas é controverso, por exemplo, se a as algas respondem ao aumento da temperatura com a síntese desse composto. Liss \& Lovelock (2007) afirmam que embora os produtos da oxidação do DMS sejam mal quantificados, eles são parte do conjunto de substâncias, algumas das quais originadas biologicamente, que atuam como NCN na atmosfera; mas são cautelosos em aceitar a existência do mecanismo de retroalimentação postulado pela hipótese CLAW e em definir se o sinal do mecanismo cibernético é positivo ou negativo. Os resultados de Foley et al. (1991), Berresheim et al. (1993), Sciare et al. (2000), Gunson et al. (2006) e Kump \& Pollard (2008) corroboram a hipótese original de um elo de retroalimentação negativo, mas os resultados de van Rijssel \& Gieskes (2002), por exemplo, contrariam. Essa situação se deve às muitas questões que ainda existem em aberto na comunidade científica sobre os processos de química atmosférica e microfísica de nuvens na interface oceano-atmosfera. Também há muitas questões em aberto sobre como a ecologia marinha influencia a abundância e a especiação do fitoplâncton e de que modo essas variáveis se ligam à liberação de DMS. Tais lacunas não devem, contudo, surpreender-nos, na medida em que a área de pesquisas na qual se insere a hipótese é muito recente. Além do mais, é importante notar que dúvidas são parte natural do processo de produção do conhecimento científico.

A hipótese CLAW tem suscitado uma intensa discussão desde que foi proposta, particularmente devido às suas implicações para o problema do aquecimento global (Schwartz 1988, Andreae \& Crutzen 1997, Gabric et al. 2001, van Rijssel \& Gieskes 2002, Ayers \& Cainey 2007, Vallina et al. 2007). Após a publicação original de Charlson et al. (1987), mais de 1.500 publicações foram feitas sobre a hipótese CLAW (Ayers \& Cainey 2007), de modo que não é exagero dizer que ela causou uma pequena revolução na biogeoquímica. A comunidade científica vem reconhecendo a importância da contribuição de Charlson, Lovelock, Andreae e Warren desde aquela publicação, o que é ilustrado pelo fato deles terem sido laureados, em 1988, com o prêmio Norbert Gerbier-Mumm, uma honraria importante concedida pela Organização Meteorológica Mundial, vinculada à $\mathrm{ONU}$, como reconhecimento por suas descobertas e pela elaboração de um mecanismo para o ciclo do enxofre (WORLD METEOROLOGICAL ORGANIZATION 2007). Além disso, o periódico Environmental Chemistry publicou recentemente um número quase exclusivamente dedicado à hipótese CLAW, tratando de suas implicações para a compreensão do sistema Terra, mas também de sua 
história, de suas controvérsias e dos avanços teóricos e empíricos relacionadas a ela (Cainey 2007).

Apesar de sua considerável aceitação pela comunidade científica e de seu papel potencial na compreensão do sistema Terra, a hipótese CLAW ainda não pôde ser adequadamente testada, pois os mecanismos de retroalimentação envolvendo processos atmosféricos e oceânicos são bastante complexos e vários aspectos da oxidação do DMS na atmosfera não são ainda bem compreendidos (Andreae \& Crutzen 1997, Ravishankara et al. 1997, Malin 2006, Ayers \& Cainey 2007, Vallina et al. 2007). Entendemos que fazendo ou não parte de um mecanismo de controle do clima, há que se considerar, de acordo com Ayers \& Cainey (2007), que tanto microalgas quanto macroalgas exercem influência no sistema climático da Terra por aumentar, na atmosfera, a concentração dos gases precursores que originam novas partículas nucleadoras de nuvens.

Está claro que as partículas de aerossol cumprem papel crucial tanto na condensação quanto na formação de nuvens, o que está de acordo com os resultados das análises de Christner et al. (2008) sobre as concentrações de nucleadores biológicos de gelo (IN, biological ice nucleators) em amostras de núcleos de gelo (fresh snowfalls) coletados na Antártica. Os achados de Christner et al. (2008) indicam que os aerossóis produzidos biologicamente (principalmente por microorganismos) são ubíquos e constituem as principais fontes de partículas ativas que induzem a formação de gelo e a precipitação quando a temperatura mínima das nuvens é alta (entre -7 e $\left.-4^{\circ} \mathrm{C}\right)$.

A esta altura, cabe enfatizar a pertinência de um aspecto epistemológico da nossa análise. Se a hipótese CLAW será mantida ou refutada é uma questão secundária para o nosso argumento e será resolvida ao seu próprio tempo pela comunidade científica. $\mathrm{O}$ que é central para nosso argumento é a capacidade das propostas de Lovelock et al. (1972) e Charlson et al. (1987) de gerar novas previsões e novas questões de pesquisa, ou seja, seu valor heurístico e sua fundamentação epistemológica. Dito de outro modo, o nosso argumento central diz respeito à cientificidade e, portanto, à legitimidade epistemológica da hipótese no âmbito do conhecimento científico estabelecido, não à sua veracidade ou falsidade. A discussão de aspectos epistemológicos do conhecimento científico precede logicamente a discussão de aspectos propriamente científicos (geralmente, empíricos), na medida em que a epistemologia toma como objeto as próprias condições de possibilidade do conhecimento científico.

Usando a terminologia da teoria da ciência do filósofo Imre Lakatos (1978), podemos dizer que houve uma alteração progressiva do problema que se buscava resolver dentro do programa de pesquisa Gaia. Isto, de acordo com Lakatos, estabelece a cientificidade do programa de pesquisa. Um esforço de pesquisa que se iniciou buscando um intermediário para o ciclo do enxofre não apenas indicou com sucesso qual seria esse composto, mas acabou por levar à descoberta de uma relação nova, algo inusitada, entre o metabolismo das algas e a cobertura de nuvens sobre os oceanos, com implicações importantes para a compreensão do clima da Terra. É necessário ter em mente que a visão dominante anterior afirmava que uma fonte puramente físico-química poderia produzir o intermediário desconhecido do ciclo do enxofre, no caso, $\mathrm{o}_{2} \mathrm{~S}$. Porém, diferentemente das fontes não-biológicas, os seres vivos produzem compostos de enxofre e os liberam continuamente (Lovelock et al. 1972, Charlson et al. 1987, Lovelock 1991). As principais fontes não-biológicas de enxofre, excluindo as atividades industriais humanas, são os vulcões e as fumarolas, que liberam $\mathrm{H}_{2} \mathrm{Se} \mathrm{SO}_{2}$. Mas as emissões vulcânicas são esporádicas, de importância secundária para o ciclo do enxofre (Charlson et al. 2000), sendo responsáveis apenas por cerca de 10 a $20 \%$ do fluxo natural global de compostos de enxofre para a atmosfera. Além disso, a liberação de gases por essas fontes não-biológicas é altamente variável no espaço e no tempo, sendo que pequenas erupções vulcânicas geralmente são de importância apenas local. Por sua vez, grandes erupções vulcânicas, que tendem a influenciar áreas maiores, são eventos muito raros (Charlson et al. 1987, Charlson et al. 2000).

A descoberta do DMS foi possível devido à adoção de certos princípios teórico-metodológicos, como a tese de que os organismos vivos são fontes de muitos compostos importantes nos ciclos dos elementos químicos. Esta é uma conseqüência da idéia de que os processos biológicos são fundamentais para a existência da combinação bastante improvável de gases na atmosfera terrestre (incluindo $\mathrm{O}_{2}, \mathrm{~N}_{2}, \mathrm{CH}_{4}$ e $\mathrm{CO}_{2}$ ), a qual não pode ser explicada recorrendo 
apenas a processos físico-químicos. A hipótese CLAW não apenas representa um novo mecanismo para a biogeoquímica do enxofre, mas sugere fortemente inovações teórico-metodológicas para o estudo integrado dos ciclos biogeoquímicos, dando particular atenção para as interações entre os organismos e seu meio material. Por exemplo, o DMS é um composto que une o ciclo da água (dado que o vapor d'água se agrega aos NCN de modo a formar nuvens) ao ciclo do enxofre, por participar simultaneamente desses dois ciclos. Além disso, como o DMS atua na formação de nuvens e, possivelmente, na conseqüente redução da temperatura da superfície planetária, ele se apresenta como um elemento potencialmente importante na determinação do clima, sobretudo quando existe uma tendência de aquecimento provocada pelo acúmulo e pela constante liberação de gases de efeito estufa (IPCC 2007). Uma abordagem que busque integrar os ciclos biogeoquímicos numa grande rede dinâmica global se mostra, assim, crítica para o entendimento do complexo processo de mudança climática em curso. A respeito disso, Cropp \& Norbury (2007) enfatizam que é uma condição necessária para avaliar o impacto do mecanismo CLAW no clima global entender os fundamentos da vida nos oceanos, mais especificamente considerar nos modelos as variações temporais e espaciais das populações de fitoplâncton. A regulação da temperatura planetária (a principal variável física modificada com as mudanças climáticas) é uma propriedade sistêmica e não apenas de partes do sistema Terra. Esta é uma importante lição metodológica a ser extraída da tradição de pesquisa que se inicia com a proposta da hipótese CLAW.

\section{IMPLICAÇÕES DA HIPÓTESE CLAW PARA A COMPREENSÃO DO CLIMA GLOBAL}

Lovelock \& Rapley (2007) propuseram recentemente, em carta à Nature, um mecanismo de geoengenharia visando mitigar os efeitos do aquecimento global. Esse mecanismo está fundamentado na contribuição do fitoplâncton para a liberação de DMS e captura de $\mathrm{CO}_{2}$ atmosférico. Os autores propõem a instalação nos oceanos de tubos ou canos verticais, flutuantes ou amarrados, com o objetivo de "aumentar a mistura de águas ricas em nutrientes abaixo da termoclina [região de transição de temperatura entre a superfície oceânica e o oceano profundo] com as águas relativamente estéreis da superfície oceânica" (Lovelock \& Rapley 2007, p. 403). Esses canos deveriam ter de 100 a 200 metros de comprimento e 10 metros de diâmetro e bombeariam água do fundo para a superfície, fornecendo, assim, nutrientes para as algas acima da termoclina. A oferta extra de nutrientes aumentaria a taxa de crescimento das algas planctônicas e, conseqüentemente, a taxa fotossintética, que está relacionada à taxa de captura de $\mathrm{CO}_{2}$ da atmosfera. Além disso, as algas aumentariam a emissão de DMS, contribuindo, em última instância, para o resfriamento do planeta, porque os produtos de sua oxidação atuam como NCN. Com efeito, conforme sugeriram Jones \& Gabric (2006), o DMS pode ser tratado - se a hipótese CLAW estiver correta - como um 'gás estufa negativo' e a duplicação de sua quantidade na atmosfera poderia levar a um resfriamento da superfície planetária em até $1,3{ }^{\circ} \mathrm{C}$. Ainda de acordo com eles, o DMS preso em bolhas de ar no gelo seria liberado para a atmosfera com o derretimento da calota antártica, o que constitui uma interessante hipótese a ser testada sobre os efeitos do DMS no clima antártico.

Este é um mecanismo similar ao proposto pela hipótese da arma dos clatratos (ou hidratos) de metano (cf. Chakoumakos 2004). Entretanto, diferentemente do metano que, uma vez liberado, aceleraria o derretimento do gelo nos pólos, aumentando a temperatura planetária, o DMS - por ser um gás estufa negativo - seria parte de um processo de retroalimentação negativa e diminuiria a temperatura planetária, contrabalançando o efeito aquecedor dos gases de efeito estufa. Por isso, segundo Lovelock \& Rapley (2007), os riscos não seriam tão grandes frente aos possíveis benefícios. Para eles, a solução desse problema envolve a remoção de 500 gigatoneladas de $\mathrm{CO}_{2}$ atmosférico, mas as tecnologias atualmente disponíveis não dão conta dessa tarefa. Eles sugerem então que sua proposta de geoengenharia induziria o sistema a se regenerar.

A proposta de Lovelock \& Rapley (2007) foi comentada por Shepherd et al. (2007), em carta publicada na edição seguinte da Nature. Esses autores advertem que aquele mecanismo de geoengenharia proposto poderia causar problemas, em vez de curar (Shepherd et al. 2007). Essa opinião contrária encontra apoio em, pelo menos, um trabalho (Arrigo 2007) que mostra importantes resultados inesperados: 
apesar do aumento da biomassa dos produtores, não ocorre seqüestro de carbono no oceano profundo, porque as partículas que carregam o carbono fixado são rapidamente degradadas pela respiração dos organismos marinhos e remineralizadas dentro dos limites das águas oceânicas superiores. Assim, é provável, segundo Shepherd et al. (2007), que quase todo $\mathrm{o} \mathrm{CO}_{2}$ capturado pelas algas seja devolvido à atmosfera no período de um ano. Além disso, o fato de interferir-se num ecossistema ainda pouco conhecido poderia gerar danos, alterando-se sua estrutura trófica, de modo que os problemas resultantes poderiam superar os possíveis benefícios daquele mecanismo de geoengenharia (para uma análise detalhada de questões éticas suscitadas pelas mudanças climáticas, ver Garvey 2008).

Nós sabemos, no entanto, que intervenções no sistema Terra como a proposta por Lovelock \& Rapley (2007) não são a única maneira pela qual os estudos na área da conexão algas-nuvens podem contribuir para mitigar os efeitos das mudanças climáticas. Há outra implicação desses estudos — de fato mais interessante - que deve ser mais explorada pelos cientistas que constroem modelos climáticos. A hipótese CLAW e os estudos que vêm sendo desenvolvidos a partir da teoria Gaia desde a década de 1990 apontam para a notável complexidade dos ciclos biogeoquímicos, bem como para o fato de que os seres vivos não devem figurar como personagens secundários nos modelos climáticos, mas sim como elementos críticos para o entendimento da dinâmica global dos elementos. As algas, por exemplo, são sorvedouros de $\mathrm{CO}_{2} \mathrm{e}$ fontes de DMS e $\mathrm{O}_{2}$ - substâncias importantes para a dinâmica climática no planeta. Os modelos climáticos que não levam em conta o papel das algas planctônicas, para usar um exemplo, são apenas representações muito simplificadas do sistema de interações desses organismos na ecologia marinha. Estamos de acordo com Cropp \& Norbury (2007) quanto à idéia de que a compreensão de sistemas complexos adaptativos, como os ecossistemas planctônicos, requer o uso de modelos baseados em processos. Os trabalhos de modelagem climática têm apontado na direção de incorporar adequadamente o papel dos organismos vivos, porém ainda o fazem de modo incipiente. Como Ayers \& Cainey (2007, p. 370) acentuam, o relatório do Grupo de Trabalho 1 do Painel Intergovernamental sobre Mudanças Climáticas (IPCC), publicado em fevereiro de 2007, não dá a devida importância à hipótese CLAW (ver IPCC 2007). Mas deveria, pois ela se pronuncia sobre (e conecta) elementos do sistema Terra cruciais para a compreensão do clima. Como apontaram Ayers \& Cainey (2007, p. 370), a menos que compreendamos muito bem as conexões entre os componentes biológicos, físicos e químicos propostos na hipótese CLAW, nós seremos incapazes de avaliar adequadamente os riscos da mudança climática para os sistemas marinhos, ou ainda de considerar estratégias apropriadas de mitigação ou adaptação.

\section{CONCLUSÕES}

Esperamos com este trabalho ter mostrado a importância dos estudos das conexões entre os organismos planctônicos da biota marinha e as nuvens para a compreensão de um importante processo na ciclagem do enxofre e no clima global. A hipótese CLAW e a área de pesquisas por ela gerada apontam para lições importantes a respeito do modo como se faz ciência atualmente, quando se estudam as mudanças climáticas. Essa hipótese oferece uma abordagem integrada dos fenômenos. Diante da crise ambiental contemporânea, uma abordagem desta natureza é imprescindível, uma vez que fenômenos de mudanças climáticas não são espacialmente localizados, mas ocorrem ao nível do sistema Terra como um todo.

Os modelos climáticos são a base para muitas ações sociopolíticas e, por isso mesmo, julgamos que tão mais acertadas serão as decisões e ações quanto melhor se apoiarem em modelos robustos que, além dos processos físicos e químicos, considerem as atividades da biota aquática e terrestre sobre aqueles processos. Essa é uma orientação heuristicamente poderosa da CST, a qual tem recebido uma contribuição bastante significativa da hipótese CLAW e dos estudos contemporâneos sobre as conexões entre a biota planctônica marinha, as nuvens e o clima global.

O desenvolvimento da tradição de pesquisa iniciada por CLAW pode oferecer contribuições relevantes para a tarefa de integração da Ecologia, atualmente um tema que ocupa tanto ecólogos quanto filósofos. Como argumentam Pickett et al. (2007), o imenso progresso das subdisciplinas da Ecologia 
acabou gerando um produto colateral indesejável: lacunas no conhecimento ecológico, manifestado pela falta de integração entre as subdisciplinas. $\mathrm{O}$ aumento de integração - sobretudo por meio da construção de teorias - é um fator necessário para aumentar a compreensão na Ecologia e também para o manejo de sistemas ecológicos (Pickett et al. 2007). Na medida em que a hipótese CLAW e a tradição de pesquisa por ela iniciada integram diferentes especialidades científicas, como Ecologia marinha, Fisiologia celular, Geoquímica, Climatologia, entre outras, a análise da sua história, dos seus fundamentos teóricos e epistemológicos pode contribuir para o trabalho de integração da própria Ecologia.

AGRADECIMENTOS: N.F.N.N. agradece ao CNPq pela bolsa de mestrado concedida durante a realização deste trabalho e à FAPESB, por financiamento de projeto de pesquisa e pela concessão de bolsa de doutorado. R.S.C. agradece ao CNPq por bolsa de iniciação científica e à FAPESB pela concessão de bolsa de mestrado. C.N.E.H. agradece ao CNPq por bolsas de produtividade em pesquisa, à FAPESB, FINEP e ao $\mathrm{CNPq}$, por financiamentos de projetos de pesquisa.

\section{REFERÊNCIAS}

ANDREAE, M.O. \& CRUTZEN, P.J. 1997. Atmospheric aerosols: biogeochemical sources and role in atmospheric chemistry. Science, 276: 1052-1058.

ANDREAE, M.O. \& RAEMDONCK, H. 1983. Dimethyl Sulfide in the surface ocean and the marine atmosphere: a global view. Science, 221: 744-747.

ARRIGO, K.R. 2007. Carbon Cycle: marine manipulations. Nature, 450: 491-492.

AYERS, G.P. \& CAINEY, J.M. 2007. The CLAW hypothesis: a review of the major developments. Environmental Chemistry, 4: 366-374.

BARNARD, W.R.; ANDREAE, M.O. \& WATKINS, W.E. 1982. The flux of dimethylsulfide from the oceans to the atmosphere. Journal of Geophysical Research, 87: 8787-8793.

BELL, A. 1986. The algae-cloud connection. ECOS Magazine, 47: 9-11.

BERRESHEIM, H.; EISELE, F.L.; TANNER, D.J.; MCINNES, L.M.; RAMSEY-BELL, D.C. \& COVERT, D.S. 1993. Atmospheric sulfur chemistry and cloud condensation nuclei
(CCN) concentrations over the northeastern pacific coast. Journal of Geophysical Research, 98: 701-12, 711.

BIGG, E.K.; GRAS, J.L. \& EVANS, C. 1984. Origin of Aitken Particles in remote regions of the southern hemisphere. Journal of Atmospheric Chemistry, 1: 203-214.

BOPP, L.; AUMONT, O.; BELVISO, S. \& MONFRAY, P. 2003. Potential impact of climate change on marine dimethyl sulphide emissions. Tellus B, 55: 11-22.

BRATBAK, G.; EGGE, J.K. \& HELDAL, M. 1993. Viral mortality of the marine alga Emiliania huxleyi (Haptophyceae) and termination of algal blooms. Marine Ecology Progress Series, 93: 39-48.

BROWN, C.W. \& YODER, J.A. 1994. Coccolithophorid blooms in the global ocean. Journal of Geophysical Research, 99: 74677482 .

CAINEY, J.M. 2007. Investigating the current thinking on the CLAW Hypothesis. Environmental Chemistry, 4: 365.

CAINEY, J.M.; SIEVERING, H. \& AYERS, G.P. 2007. Where to now? A synthesis of current views of the CLAW hypothesis. Environmental Chemistry, 4: 406-409.

CARMO, R.S.; NUNES-NETO, N.F. \& EL-HANI, C.N. 2009. Gaia Theory in Brazilian High School Biology Textbooks. Science \& Education, 18: 469-501.

CHAKOUMAKOS, B. 2004. Preface to the Clathrate Hydrates special issue. American Mineralogist, 89: 1153-1154.

CHARLSON, R.J.; LOVELOCK, J.E.; ANDREAE, M.O. \& WARREN, S.G. 1987. Oceanic phytoplankton, atmospheric sulphur, cloud albedo and climate. Nature, 326: 655-661.

CHARLSON, R.J.; ANDERSON, T.L. \& McDUFF, R.E. 2000. The sulfur cycle. Pp 343-359. In: M.C. Jacobson, R.J. Charlson, H. Rodhe \& G.H. Orians (eds.). Earth System Science: from biogeochemical cycles to global changes. Elsevier, San Diego. $527 \mathrm{p}$.

CHRISTNER, B.C.; MORRIS, C.E.; FOREMAN, C.M.; CAI, R. \& SANDS, D.C. 2008. Ubiquity of biological ice nucleators in snowfall. Science, 319: 1214.

CLARKE, A.D.; KAPUSTIN, V.N.; EISELE, F.L.; WEBER, R.J. \& McMURRY, P.H. 1999. Particle production near marine clouds: sulfuric acid and predictions from classical binary nucleation. Geophysical Research Letters, 26: 2425-2428.

CROPP, R. \& NORBURY, J. 2007. Plankton modelling and CLAW. Environmental Chemistry, 4: 388-390. 
DeBOSE, J.L.; LEMA, S.C. \& NEVITT, G.A. 2008 Dimethylsulfoniopropionate as a foraging cue for reef fishes. Science, 319: 1356.

FOLEY, J.A.; TAYLOR, K.E. \& GHAN, S.J. 1991. Planktonic dimethylsulfide and cloud albedo: an estimate of the feedback response. Climatic Change, 18: 1-15.

FUHRMAN, J.A. 1999. Marine viruses and their biogeochemical and ecological effects. Nature, 399: 541.

GABRIC, A.; GREGG, W.; NAJJAR, R.G.; ERICKSON, D. J. III. \& MATRAI, P. 2001. Modeling the biogeochemical cycle of dymethylsulfide in the upper ocean: a review. Chemosphere Global Change Science, 3: 377-392.

GABRIC, A.J.; SIMÓ, R.; CROPP, R.A.; HIRST, A.C. \& TOTTERDELL, I.J. 2004. Modeling estimates of the global emissions of dimethylsulfide under enhanced greenhouse conditions. Global Biogeochemical Cycles, 18: GB2041.

GARVEY, J. 2008. The Ethics of Climate Change: Right and Wrong in a Warming World. Continuum, London. 179 p.

GUNSON, J.R.; SPAL, S.A.; ANDERSON, T.R.; JONES, A.; TOTTERDELL, I.J. \& WOODAGE, M.J. 2006. Climate sensitivity to ocean dimethylsulphide emissions. Geophysical Research Letters, 33: L07701.

HAMILTON, W.D. \& LENTON, T.M. 1998. Spora and Gaia: how microbes fly with their clouds. Ethology, Ecology \& Evolution, 10: $1-16$.

IPCC (INTERGOVERNMENTAL PANEL ON CLIMATE CHANGE). 2007. Climate Change, 2007: The Physical Science Basis. Contribution of Working Group I to the Fourth Assessment Report of the Intergovernamental Panel on Climate Change. Cambridge University Press, Cambridge and New York. 996p.

HUEBERT, B. 2007. Do I believe in CLAW? Environmental chemistry, 4: 375-376.

JACOBSON, M.C.; CHARLSON, R.J.; RODHE, H. \& ORIANS, G.H. 2000. Earth System Science: from biogeochemical cycles to global changes. Elsevier, San Diego. 527p.

JONES, G. \& GABRIC, A. 2006. Sulphur aerosols released from melting sea ice may influence Antarctic climate. Australian Antarctic Magazine, 10: 28-29.

KASTING, J.F. \& SIEFERT, J.L. 2002. Life and the evolution of Earth's atmosphere. Science, 296: 1066-1068.

KELLER, M.D. 1991. Dimethyl sulfide production and marine phytoplankton: the importance of species composition and cell size. Biological Oceanography, 6: 375-382.
KIENE, R.P.; WILLIAMS, L.P.H. \& WALKER, J.E. 1998. Seawater microorganisms have a high affinity glycine betaine uptake system which also recognizes dimethylsulfoniopropionate. Aquatic Microbial Ecology, 15: 39-51.

KIRST, G.O. 1996. Osmotic adjustment in phytoplankton and macroalgae: the use of dimethylsulfoniopropionate (DMSP). Pp 121-129. In: R.P. Kiene, P.T. Visscher, M.D. Keller \& G.O. Kirst (eds.). Biological and Environmental Chemistry of DMSP and Related Sulfonium Compounds. Plenum Press, New York. 430p.

KUMP, L.R.; KASTING, J.F. \& CRANE, R.G. 1999. The Earth System. Prentice Hall, New Jersey. 200p.

KUMP, L.R. \& POLLARD, D. 2008. Amplification of Cretaceous warmth by biological cloud feedbacks. Science, 320: 195.

LAKATOS, I. 1978. The Methodology of Scientific Research Programmes. Pp. 8-93. In: J. Worrall \& G. Currie (eds.). Philosophical Papers Volume 1. Cambridge University Press, Cambridge. 250p.

LISS, P.S.; MALIN, G. \& TURNER, S.M. 1993. Production of DMS by phytoplankton. Pp. 1-14. In: G. Restelli \& G. Angeletti (eds.). Dimethylsulphide: oceans, atmosphere and climate. Kluwer Academic, Dordrecht. 393p.

LISS, P. \& LOVELOCK, J. 2007. Climate change: the effect of DMS emissions. Environmental Chemistry, 4: 377-378.

LECK, C. \& BIGG, E.K. 2007. A modified aerosol-cloud-climate feedback hypothesis. Environmental chemistry, 4: 400-403.

LEVIT, G.S. \& KRUMBEIN, W.E. 2000. The biosphere-theory of V.I. Vernadsky and the Gaia-theory of James Lovelock: a comparative analysis of the two theories and traditions. Journal of General Biology, 61: 133-144.

LOVELOCK, J.E.; MAGGS, R.J. \& RASMUSSEN, R.A. 1972. Atmospheric dimethyl sulphide and the natural sulphur cycle. Nature, 237: 452-453.

LOVELOCK, J.E. 1988. The ages of Gaia: a biography of our living Earth. W. W. Norton \& Co, New York. 255p.

LOVELOCK, J.E. 1991. Gaia: the Practical Science of Planetary Medicine. Oxford University Press, Oxford. 192p.

LOVELOCK, J.E. 1997. A geophysiologist's thoughts on the natural sulphur cycle. Philosophical Transactions of the Royal Society of London, 352: 141.

LOVELOCK, J.E. \& RAPLEY, C. 2007. Ocean pipes could help the Earth to cure itself. Nature, 449: 403.

MALIN, G. 2006. New pieces for the marine sulfur cycle jigsaw. Science, 314: 607-608. 
NELSON, D.L. \& COX, M.M. 2009. Lehninger Principles of Biochemistry (Fifth Edition). W.H. Freeman, New York. 1100p. NUNES-NETO, N.F. 2008. Bases Epistemológicas para um Modelo Funcional em Gaia. Dissertação de Mestrado. Programa de Pós-Graduação em Ensino, Filosofia e História das Ciências da Universidade Federal da Bahia e Universidade Estadual de Feira de Santana, Salvador, Brasil. 187p.

PICKETT, S.T.A.; KOLASA, J. \& JONES, C.G. 2007. Ecological understanding: The nature of theory and the theory of nature. Elsevier, San Diego. 233p.

PROCTOR, L.M. \& FUHRMAN, J.A. 1991. Roles of viral infection in organic particles flux. Marine Ecology Progress Series, 69: 133-142.

PROCTOR, L.M. 1997. Advances in the study of marine viruses. Microscopy Research and Technique, 37: 136-161.

RAVISHANKARA, A.R.; RUDICH, Y.; TALUKDAR, R. \& BARONE, S.B. 1997. Oxidation of atmospheric reduced sulphur compounds: perspective from laboratory studies. Philosophical Transactions of the Royal Society of London, 352: 171-182.

SCHWARTZ, S.E. 1988. Are global cloud albedo and climate controlled by marine phytoplankton? Nature, 336: 441-445.

SCIARE, J.; MIHALOPOULOS, N. \& DENTENER, F.J. 2000. Interannual variability of atmospheric dimethylsulfide in the southern Indian Ocean. Journal of Geophysical Research, 105: 26369-26377.

SHAW, G.E. 1983. Bio-controlled thermostasis involving the sulfur cycle. Climatic change, 5: 297-303.

SHAW, G.E. 2007. Do biologically produced aerosols really modulate climate? Environmental chemistry, 4: 382-383.

SHEPHERD, J.; IGLESIAS-RODRIGUEZ, D. \& YOOL, A. 2007. Geo-engineering might cause, not cure, problems. Nature, 449: 781.

SIMÓ, R. 2001. Production of atmospheric sulfur by oceanic plankton: biogeochemical, ecological and evolutionary links. Trends in Ecology \& Evolution, 16: 287-294.

SIMÓ, R. \& PEDRÓS-ALIÓ, C. 1999. Role of vertical mixing in controlling the oceanic production of dimethyl sulphide. Nature, 402: 396-399.

SMETACEK, V. \& CLOERN, J.E. 2008. On phytoplankton trends. Nature, 319: 1346-1348.

SPIESE, C.E.; KIEBER, D.J.; NOMURA, C.T. \& KIENE, R.P. 2009. Reduction of dimethylsulfoxide to dimethylsulfide by marine phytoplankton. Limnology and Oceanography, 54: 560570.
SUNDA, W.; KIEBER, D.J.; KIENE, R.P. \& HUNTSMAN, S. 2002. An antioxidant function for DMSP and DMS in marine algae. Nature, 418: 317-320.

SUTTLE, C.A.; CHAN, A.M. \& COTTRELL, M.T. 1991. Use of ultrafiltration to isolate viruses from seawater which are pathogens of marine phytoplankton. Applied and Environmental Microbiology, 57: 721-726.

STALLARD, R.F. 2000. Tectonic processes and erosion. Pp195229. In: M.C. Jacobson, R.J. Charlson, H. Rodhe \& G.H. Orians (eds.). Earth System Science: from biogeochemical cycles to global changes. Elsevier, San Diego. 527p.

STEFELS, J. 2000. Physiological aspects of the production and conversion of DMSP in marine algae and higher plants. Journal of Sea Research, 43: 183-197.

STEINKE, M.; MALIN, G. \& LISS, P.S. 2002. Trophic interactions in the sea: an ecological role for climate relevant volatiles? Journal of Phycology, 38: 630-638.

TWOMEY, S. 1977. The influence of pollution on the shortwave albedo of clouds. Journal of the atmospheric sciences, 34: 11491152 .

VAN RIJSSEL, M. \& GIESKES, W. 2002. Temperature, light, and the dimethylsulfoniopropionate (DMSP) content of Emiliania huxleyi (Prymnesiophyceae). Journal of Sea Research, 48:17-27.

VALLINA, S.M.; SIMÓ, R.; GASSÓ, S.; BOYER-MONTÉGUT, C.; DEL RÍO, E.; JURADO, E. \& DACHS, J. 2007. Analysis of a potential "solar radiation dose-dimethylsulfide-cloud condensation nuclei" link from globally mapped seasonal correlations. Global Biogeochemical Cycles, 21: 1-16.

VERNADSKY, V.I. 2007. Geochemistry and The Biosphere: essays by Vladimir I. Vernadsky, Synergetic Press, Santa Fé, New Mexico. 427p.

WATSON, A. \& LOVELOCK, J.E. 1983. Biological homeostasis of the global environment: the Parable of Daisyworld. Tellus B, 35: 284-289.

WORLD METEOROLOGICAL ORGANIZATION. Winners of the Norbert Gerbier-Mumm International Award. http://www. wmo.ch/pages/about/awards/winners_mumm.html. (Acesso em: 20/10/2007).

WOLFE, G.V.; STEINKE, M. \& KIRST, G.O. 1997. Grazingactivated chemical defence in a unicellular marine alga. Nature, 387: 894-897.

Submetido em 22/06/2009.

Aceito em 19/10/2009. 